\title{
Dynamic Admission Control for Quality of Service in IP Networks
}

\author{
J. Kasigwa, V. Baryamureeba, and D. Williams
}

\begin{abstract}
The goal of admission control is to support the Quality of Service demands of real-time applications via resource reservation in IP networks. In this paper we introduce a novel Dynamic Admission Control (DAC) mechanism for IP networks. The DAC dynamically allocates network resources using the previous network pattern for each path and uses the dynamic admission algorithm to improve bandwidth utilization using bandwidth brokers. We evaluate the performance of the proposed mechanism through trace-driven simulation experiments in view point of blocking probability, throughput and normalized utilization.
\end{abstract}

Keywords-Bandwidth broker, dynamic admission control (DAC), IP networks, quality of service, real-time flows.

\section{INTRODUCTION}

$\mathrm{A}$ DMISSION control in IP networks has become an important area of interest for most network researchers and network managers in the provisioning of end-to-end Quality of Service (QoS) [1], [2]. Admission control is a key component for QoS delivery in IP networks because it determines the extent to which network resources are utilized and whether the contracted QoS requirements are actually delivered.

Although significant progress has been made in providing scalable QoS guarantees to a wide range of real-time applications, little attention has been paid to the dynamic aspect of admission control in IP networks [3]. Current research has ignored an important issue of admission control in IP networks, particularly when traffic is traversing multiple IP network domains [4]. Real-time applications need more stringent QoS requirements in terms of bandwidth, latency and loss. QoS does not create bandwidth, but manages it more effectively to meet the wide application requirements [5].

To satisfy these requirements the IETF proposed Differentiated Service (DiffServ) architecture in the end of 1990. DiffServ provides a simple and coarse method of classifying services of various applications. There are currently three Per Hop Behaviors (PHB) defined that effectively represent three service classes such as Expedited Forwarding (EF), Assured Forwarding (AF) and Best Effort (BE). DiffServ simplifies the function of core nodes and distributes the complex control function to edge nodes to provide scalability [6]. The bandwidth broker is introduced for the control and management of end-to-end QoS to reduce the complexity of QoS control plane [7].

The static admission control methods such as PBAC and MBAC operate well in the management of simple IP networks However, the static admission control methods cannot cope with dynamic changes of input traffic and network topology since it reflects the IP network status. In this respect, the bandwidth broker model provides the dynamic provisioning.
The dynamic admission control allocates the network resources to the requested user flow according to the network situation. However, this mechanism also introduces its own scalability issue to handle large volumes of flows as the network system scales [8][9].

In this paper, we propose the Dynamic Admission Control (DAC) mechanism. The DAC allocates the network resource using the previous traffic pattern to each path. Edge nodes do not need communication with the bandwidth broker if the allocated bandwidth for the path is sufficient to satisfy the flow's request. In the proposed DAC mechanism, "dynamic" means that the bandwidth broker allocates the resource to the path dynamically and the amount of bandwidth allocated to the path is not fixed but is variable upon the traffic flow's QoS requirements.

The remainder of the paper is organized as follows. In section 2, we first summarize the previous work of the admission control. In section 3 and 4, we describe detail procedure of the DAC mechanism and present the simulation results and the performance evaluation analysis. We conclude the paper in Section 5.

\section{DyNAMIC ADMISSION CONTROL MECHANISM}

The proposed Dynamic Admission Control (DAC) mechanism in IP networks is divided into two phases: Pathlevel admission control and Link-level admission control. The bandwidth broker manages only the path-level bandwidth resources within the domain and path-level admission control is performed at the ingress edge node. For the link-level admission control, MBAC is performed for management of the link-level bandwidth along the path. For the DAC mechanism, a two-phase measurement-based admission control method is used [10].

The bandwidth broker calculates the path bandwidth within the domain and sends it to each ingress edge node. The ingress edge node then performs the path-level admission control using the path bandwidth. If the path bandwidth is fully occupied, the ingress edge node performs the movable boundary bandwidth management and extends the path bandwidth of the Expedited Forwarding (EF) or Assured Forwarding (AF) service. It also performs the link-level admission control using MBAC. On the other hand, if congestion occurs in the core node along the path, a congestion notification is sent to each ingress edge node through the bandwidth broker [11]. The ingress edge node then performs only link-level admission control using DAC if there is enough path bandwidth.

The proposed DAC framework simplifies the bandwidth calculation in the bandwidth broker by performing only pathlevel resource management. It also has an advantage in that the ingress edge node can perform the admission control for 
itself within the path-level bandwidth regardless of any communication with the bandwidth broker. Furthermore, when the path-level bandwidth allocated initially is fully occupied, the proposed mechanism performs dynamic bandwidth management with dynamic admission control to extend the allocated bandwidth at the edge node for the linklevel admission control. Another advantage of the proposed framework is that the bandwidth broker need not maintain any link-level bandwidth information for the link level admission control.

\section{DYNAMIC ADMISSION CONTROL AlgORITHM}

In this section, we describe the basic operation model and the algorithm of the proposed dynamic admission control mechanism. Dynamic admission control allocates the network resources to the requested user flow properly according to the network situation. It means that this algorithm has to react fast to the unexpected traffic pattern changes. The efficient network resource management of IP networks depend on how ingress edge node can perform the distinct functions according to service classes instead of simple operation in core node. The traffic violation information of network should be gathered immediately by the bandwidth broker and feedback given to the ingress edge node.

In the proposed DAC mechanism, the bandwidth broker initially allocates provisioned bandwidth to each path. The state of a path maintains the amount of bandwidth that has been allocated to the path. When a flow reservation set-up request along a path arrives, the ingress edge node only needs to check the corresponding path whether the amount of bandwidth allocated to the path is sufficient to satisfy the flow's request. If the answer is positive, the flow request is accepted. Only when the allocated bandwidth of the path is less than the requested bandwidth, the ingress edge node requests the additional bandwidth to the bandwidth broker. The amount of the additional bandwidth is calculated in the ingress edge node based on the previous traffic pattern information.

The bandwidth broker will check each link along the path to see whether there is available bandwidth at all the links except already allocated bandwidth for each path. If available bandwidth is sufficient, the bandwidth broker allocates the additional bandwidth to the path and informs each node along the path to change allocated bandwidth information for that path. Therefore, this mechanism simplifies the bandwidth calculation in the bandwidth broker by limiting the resource management to the only path level. That is, the bandwidth broker can minimize the communication between the bandwidth broker and each node in the domain. Also, it has some advantages that the ingress edge node performs the admission control for itself in path level, the estimation of additional bandwidth, and the dynamic bandwidth allocation.

A more formal and detailed description of the scheme is presented by the pseudo-code in Fig. 1 and Fig. 2.

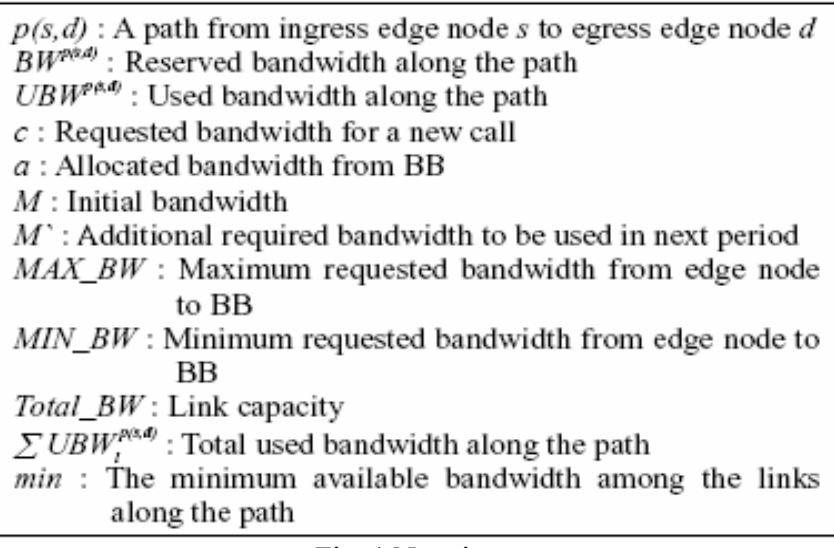

Fig. 1 Notations

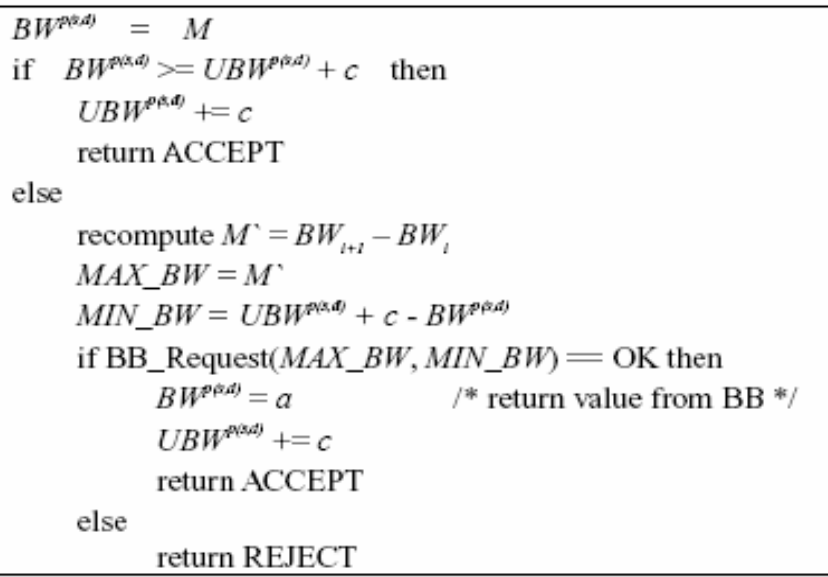

Fig. 2 Dynamic admission control at the ingress edge node

Fig. 2 describes the admission control in the ingress edge node. If the reserved bandwidth is sufficient for new flow setup request, the ingress edge node accepts the flow request. If not, the ingress edge node computes the additional bandwidth considered to be needed in next period and requests bandwidth allocation to the bandwidth broker. That additional bandwidth, $B W_{i+1}$ denotes the amount of bandwidth that the ingress edge node will request to bandwidth broker in time $T_{i+1}$, is estimated based the previous traffic pattern such as follows:

$$
B W_{i+1}=\frac{U B W_{i}-U B W_{i-1}}{T_{i}-T_{i-1}} \cdot \Delta t
$$

$B W_{i}$ is the allocated bandwidth in time $T_{i}$, and $U B W_{i}$ is the used bandwidth in time $T_{i}$.

Also, $\Delta t$ means that the average value of time interval from $T_{i-1}$ to $T_{i}$. Through this $\Delta t$, we can reflect the previous traffic patterns to the next bandwidth allocation.

$$
\Delta t=\frac{\sum_{k=0}^{i} T_{k}-T_{k-1}}{i-1}
$$

If the minimum available bandwidth among the links along the path is greater or equal to the maximum requested bandwidth 
from the edge node to the bandwidth broker, then additional bandwidth is allocated. In the case that the available bandwidth in each link along the path is sufficient to satisfy the requested additional bandwidth from the ingress edge node, the bandwidth broker will allocate $M A X \_B W$ or $\min$ to the ingress edge node as well as to each node in domain. Otherwise, the flow set-up request is rejected.

\section{PERFormanCE EVAluAtion}

To evaluate the proposed DAC mechanism, we perform extensive simulation experiments to ascertain the efficacy of the proposed solution. To measure the accuracy and effectiveness of the DAC algorithms, we simulate different admission control mechanisms and the obtained results are compared with the DAC mechanism.

\section{A. Simulation Model and Results}

In this section, we conduct simulation experiments to evaluate the accuracy of the aforementioned admission control algorithms by performing a set of admission control experiments. We used the NS-2 (network simulator-2) simulator and the peer-to-peer simulation model.

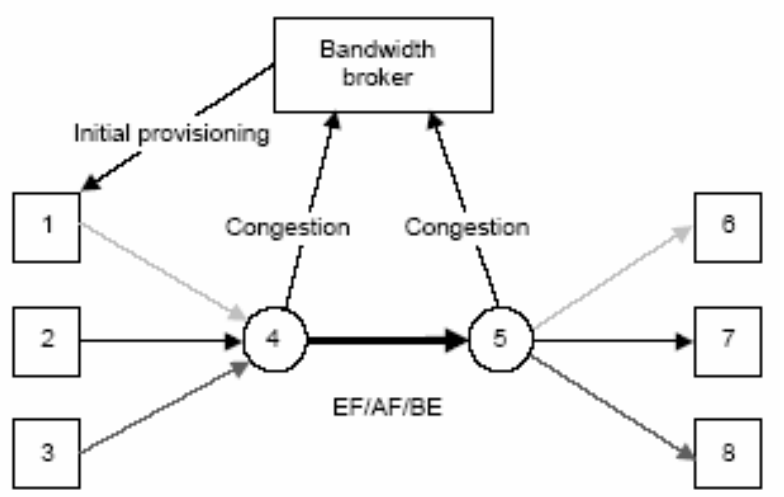

Fig. 3 Simulation model

The workload consists of a set of twenty 30-minute traces of MPEG and JPEG compressed video of Expedited Forwarding (EF) and Assured Forwarding (AF) traffic flows. We used the actual traces of MPEG-compressed video traffic for the experiments.

In the implementation of the proposed dynamic admission control algorithms, we perform a set of trace-driven simulation and admission control experiments for a wide variety of traffic mixes and network capacities. To achieve this, we first implement a number of admission control algorithms from the aforementioned traffic classes and determine their respective admissible regions for various traffic mixes and QoS parameters.

Each path has an initial bandwidth provisioning to $\mathrm{EF} / \mathrm{AF} / \mathrm{BE}$ traffic services. We evaluate the blocking probability of each ingress edge node and packet loss probability of the core node of DAC and compare them with the measurement-based admission control (MBAC) method.
Simulation time is always 1200 seconds of which the first 600 seconds are discarded as "warming period". The particular area of interest is the blocking probability, throughput and packet loss probability of different admission control methods. The node is loaded with exponentially distributed inter-arrival and holding times. The mean holding time is $30 \mathrm{~s}$, the mean inter-arrival time is set to achieve load factor $\rho$. We define the input traffic load factor $(\rho)=$ mean holding time/call attempt time interval. The target loss probability of the AF service is $10^{-3}$, the end-to-end delay is $50 \mathrm{~ms}$.

Our simulation scenario consists of a first-come-first-serve multiplex with buffer size $B$, MPEG and JPEG real-time traffic traces as the workload, and the link capacity of $C=45$ Mbps, unless otherwise noted. In all experiments, we set the buffer size $B$ equal to $C$ times the required delay bound so that delay violation probability is the same as packet loss probability. i.e.

Buffer size $=$ Link capacity $x$ delay bound i.e. $B=C d$

\section{B. Result Analysis}

An admission control test's effectiveness is ultimately determined by its ability to correctly decide whether or not a new traffic flow can be admitted while still satisfying the QoS constraints of all established flows plus the new flows. We calculate $95 \%$ confidence intervals for each probability estimated via simulation using the method of batch mean [12].

Fig. 4 shows the blocking probability of the proposed DAC mechanism compared to the static MBAC for $\mathrm{EF}$ and $\mathrm{AF}$ traffic services.

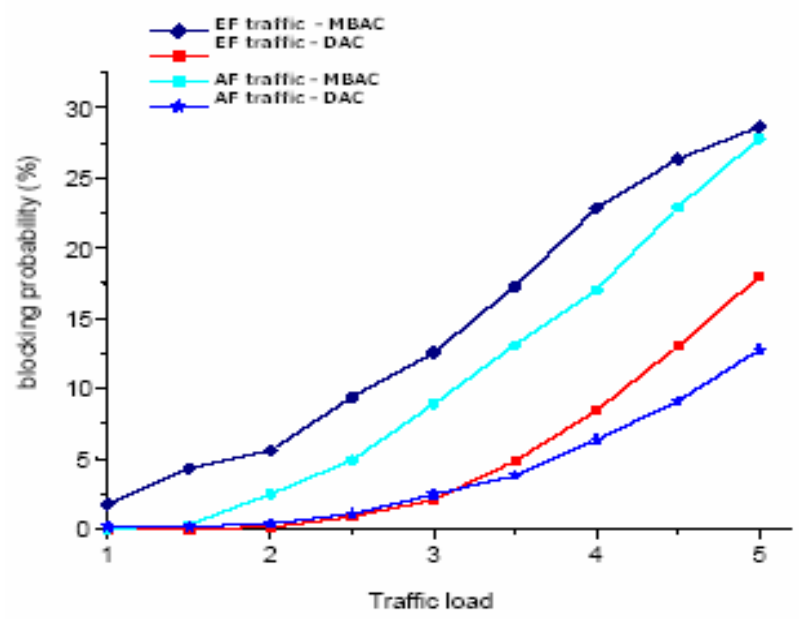

Fig. 4 Blocking probability of proposed DAC

This simulation result shows that the proposed DAC mechanism has less blocking probability than static measurement based admission control (MBAC) mechanisms even with a high traffic load. As the proposed mechanism can use the unused bandwidth of BE service through the dynamic admission control method and can accept more requested flows. On the other hand, the static admission control allocates a fixed bandwidth of the link to each service class and this allocated bandwidth is not changed. 
From the simulation experiments, we can see from Fig. 5 that AF3 connections experience higher end-to-end packet delay with MBAC as compared to the proposed DAC.



Fig. 5 AF3 traffic bottleneck link delays

DAC mechanism gives better end-to-end throughput performance for real-time applications at different packet arrival intensities. This admission control test is able to capture non-exponential relationship between packet losses and delays.

Fig. 6 shows the packet loss probability of the proposed mechanism for AF service when the traffic load is 5 and the buffer size is 500 . We can also see the effect of the admission control scheme of the proposed mechanism. If a mechanism has no proper admission control scheme, the target loss probability cannot be satisfied when traffic load is 5 .



Fig. 6 Effect of dynamic admission control

From Fig. 6, it can also be seen that even though the proposed DAC mechanism accepted more flows than static MBAC, the target loss probability $\left(10^{-3}\right)$ of the AF service is guaranteed.

\section{CONCLUSION}

In this paper, a novel Dynamic Admission Control mechanism for IP networks has been designed and developed for scalable support of end-to-end QoS guarantees in IP networks. The proposed DAC mechanism simplifies the calculation of the bandwidth data table in the bandwidth broker by performing only path-level resource management.

Both the theoretical and simulated results of the proposed DAC mechanism provide better end-to-end QoS guarantees than the existing admission controls methods. With DAC, the demands of real time traffic do not override all other traffic requirements, but rather traffic link resources are shared in a more dynamic and flexible manner.

For the performance evaluation of the proposed mechanism, we presented simulation results in terms of blocking probability, loss probability, and throughput, comparing the proposed DAC mechanism with static MBAC, comparing our mechanism with the existing dynamic provisioning mechanisms using an ns-2 simulator. Through simulation results, we proved that the proposed DAC mechanism guarantee users QoS requirements and provide bandwidth efficiency.

\section{REFERENCES}

[1] Elek, V., Karlsson, G. and Ronngren, R. (2000). Admission Control Based on End-to-End Measurements. In: Proceedings of the INFOCOM 2000, 19th Annual Joint Conference of the IEEE Computer and Communications Societies, Stockholm Sweden, 2:622-630.

[2] Samir, C., and Jongbok, B. (2002). Quest for the end-to-end network QoS. Eighth Americas Conference on Information Systems, 1919-1925.

[3] Eleni, M., Charalampos, C., Panos, G., Danny, G. and Griffin, D. (2003). Admission Control for Providing QoS in DiffServ IP Networks. The TEQUILA Approach. IEEE Communications Magazine, 38-44.

[4] Mei, Y., Yan, H., Jaime, K., Meejeong, L. Tatsuya, S. and Matsubara, D. (2004). An End-to-End QoS Framework with On-Demand Bandwidth Reconfiguration. In: Proceedings of the IEEE INFOCOM, 0-7803-8356$7 / 04$.

[5] L. Mathy, C. Edwards and D. Hutchison, The Internet: A Global Telecommunications Solution? IEEE Network Magazine, pp.46-57, July/August 2000.

[6] M. Albrecht, M. Kster, (2003). End-to-end QoS Management for Delaysensitive Scalable., The Proceedings of the 25 Annual Conf. on Local Computer Networks (LCN'03), Tampa, FL.

[7] A.Terzis, .The role of BB in Traffic Engineering. Proc. of ICNP'99, IEEE Computer Society. Sec 3.4.1.

[8] Z.-L.Zhang et al., On Scalable Design of Bandwidth Brokers, IEICE Trans. Commun., Vol.E84-B, No.8, pp.2011-2025, August 2001.

[9] Rhee, W.S, Lee, J., Yang, M., Kim, S. (2003). "Admission control mechanism using measurement based dynamic provisioning in differentiated service network," IEEE ICCS2003, Singapore.

[10] Rhee, W., Kim, H., Park, K., Kim, K., and Kim, S. (2001). Two Phase Edge-to-Edge Distributed Measurement Based Admission Control Mechanism in Large IP Networks. In: Proceedings of the IEEE GLOBECOM 2001, 2571-2575.

[11] Zhiqun, Z., Xu, S., and Wei, D. (2004). MPLS ATCC. An Active Traffic and Congestion Control Mechanism in MPLS. In: Proceedings of the International Conferences on Infotech and Infonet (ICII2004, 5(3):234241.

[12] P. Bratley, B. Fox, and L. Schrage, A Guide to Simulation. New York: Springer-Verlag, second ed., 1987. 
World Academy of Science, Engineering and Technology International Journal of Computer and Information Engineering

Vol:1, No:8, 2007

Justine Kasigwa holds a Masters Degree in Computer Science and Information Technology (2002) and Bachelor of Sciences Degree with a major in Mathematics (1999). She is currently undertaking her PhD. in Computer Science from Makerere University. Her research interests centers on Quality of Service and data security in IP networks.

Dr. Venansius Baryamureeba received his Ph.D. in Computer from the University of Bergen (UiB) in Norway 2000. He was awarded the prize of the best Ph.D. student for the year 2000 in recognition of his overall academic Excellency at UiB. His also the Director of Makerere University Institute of Computer Science and also the Chairman and Managing Director of ICT Consults Ltd., the leading ICT consulting firm in the Uganda, and several other assignments and tasks both in Government and non-government organizations.

Dr. Ddembe Williams received his $\mathrm{PhD}$ in 2003 from London South Bank University. He is the director, Centre for Advanced Systems Modelling and Simulation, Faculty of Business, Computing and Information Management, and Senior Lecturer in Decision Sciences at the same University in the UK. 